Vol 12, Issue 12, 2019

\title{
SELF-NANO EMULSIFYING DRUG DELIVERY SYSTEM OF EFAVIRENZ: FORMULATION, IN VITRO EVALUATION AND CHARACTERIZATION
}

\author{
PAMU SANDHYA* \\ Department of Pharmaceutics, Shadan Women's College of Pharmacy, Hyderabad, Telangana, India. Email: sandhyapasikanti@gmail.com \\ Received: 04 August 2019, Revised and Accepted: 24 September 2019
}

\begin{abstract}
Objective: The main objective of this study was to preparation and evaluation of efavirenz (EFV) to enhance its solubility and dissolution rate by selfemulsifying drug delivery system.
\end{abstract}

Methods: EFV self-emulsifying drug delivery systems (SNEDDS) were formulated using different oils, surfactant, and co-surfactant. Peceol, Tween 20, and Capmul MCM were used as oil, surfactant, and co-surfactant, respectively, followed by the evaluation by the performance of different tests such as visual observation, solubility studies, thermodynamic stability study, transmittance studies, drug content, and in-vitro release study.

Results: Fourier-transform infrared studies revealed negligible drug and polymer interaction. From the phase diagram, it was observed that selfemulsifying region was enhanced with increasing surfactant and co-surfactant concentrations with oil. F13 was selected as optimized formulation on the basis of physicochemical parameters, particle size, and in-vitro dissolution studies with the release of $98.39 \pm 5.10 \%$ drug in 1 hour. The optimized formulation size was found to be $156.7 \mathrm{~nm}$ as mean droplet size and Z-Average of $808.6 \mathrm{~nm}$ with $-18.3 \mathrm{mV}$ as zeta potential.

Conclusion: The study demonstrated that SNEDDS was a promising strategy to enhance the dissolution rate of EFV by improving solubility.

Keywords: Efavirenz, Antiretroviral, Self-emulsifying drug delivery systems, Peceol, Z-Average.

(C) 2019 The Authors. Published by Innovare Academic Sciences Pvt Ltd. This is an open access article under the CC BY license (http://creativecommons. org/licenses/by/4. 0/) DOI: http://dx.doi.org/10.22159/ajpcr.2019.v12i12.35227

\section{INTRODUCTION}

Self-emulsifying drug delivery systems (SNEDDS) is a potential tool for bioavailability enhancement of poor aqueous soluble drugs [1]. SNEDDS is basically an isotropic mixture of drug, oil, surfactant, and co-surfactant. Such dosage forms exhibit a characteristic feature spontaneous emulsion formation on dilution with water with little or no energy input [2]. Depending on the excipients and formulation techniques, self-micro and self-nanoemulsifying drug delivery systems (self-microemulsifying drug delivery system [SMEDDS]/SNEDDS) may be developed [3]. SMEDDS has a droplet size range of 100-250 nm and form optically clear to translucent dispersions. Contrarily, SNEDDS has a droplet size of $<100 \mathrm{~nm}[4]$.

Efavirenz (EFV) is an anti-HIV drug that acts by inhibition of nonnucleoside reverse transcriptase of HIV [5]. EFV majorly exhibits the non-competitive inhibitory activity of HIV-1 reverse transcriptase. Being a BCS class II drug, its aqueous solubility is low, whereas permeability is high that is responsible for low bioavailability [6].

In the present study, EFV would be formulated as SNEDDS, and the assessment of influence of varying ratios of oil: Smix (a mixture of surfactantand co-surfactant) in different concentrations on release of EFV would be studied. The influence of amounts of surfactant, co-surfactant, and oil on globule size, turbidly, and percentage drug release in $20 \mathrm{~min}$ was studied during the process of optimization. The formulation with all parameters optimized along with having enhanced in-vitro drug release is expected to increase oral absorption of the drug [7].

\section{MATERIALS AND METHODS FOR EFV SNEDDS}

Materials

EFV was obtained from Hetero drugs ltd, Hyderabad. Labrafac PG and Labrafil M 2125 Capmul MCM, Cremophor EL, Capyrol PGMC, Miglynol $812 \mathrm{~N}$ and Transcutol P, Acrysol K-150, Kolliphor ELP, Kolliphor HS 15, and Brji-35 were procured from Gattefosse Ltd., Mumbai; PEG 200,
PEG-600, polysorbate 20, Tween 20, Tween 80 , and Oleic acid were obtained from SDFCL, Mumbai.

\section{Methods}

Solubility studies

An excess amount ( $10 \mathrm{mg}$ ) of EFV on addition to $2 \mathrm{ml}$ of each excipient (Oils - Labrafac PG, Peceol, Acrysol k-150, Capryol PGMC, Oleic acid, and Miglynol 812N; Surfactants - Lauroglycol, Tween 20, Cremophor EL, Kolliphor ELP, and Kolliphor HS 15; Co-surfactants - PEG 200, Capryol PGE, Brji-35, Capryol 90, and Capmul MCM) were kept in mechanical shaker for $24 \mathrm{~h}$ and centrifuged at $10,000 \mathrm{rpm}$ for $20 \mathrm{~min}$ using a centrifuge. Supernatant was filtered through membrane filter using $0.45 \mu \mathrm{m}$ filter disk. The resultant solution was analyzed for ultraviolet (UV) absorbance at $248 \mathrm{~nm}$ after dilution with methanol and determination of amount of drug was done. Suitable surfactant, co-surfactant, and oil in which drug exhibited good solubility were selected by solubility studies [8].

\section{Pseudo-ternary phase diagram}

Pseudo-ternary phase diagrams have been constructed by the water titration method maintaining the temperature at $25^{\circ} \mathrm{C}$. The first step was a proper mixing of different volume ratio (1:1,2:1, and 3:1) of surfactant and co-surfactant (Smix) in each group followed by mixing of oil and surfactant/co-surfactant mixture (Smix) in variable volume ratios 1:9-9:1 (1:9, 2:8, 3:7, 4:6, 5:5, 6:4, 7:3, 8:2, and 9:1) w/w for all the three Smix ratios 1:1, 2:1, and 3:1. Titration of certain ratios of oil, surfactant, and co-surfactant mixture by drop wise addition of deionized water was performed simultaneously agitating the mixture gently. Determination of proper ratio of one excipient to another in SNEDDS formulation was made, and Chemix software was employed for the construction of pseudo-ternary plots [9].

\section{Visual observation}

About $0.2 \mathrm{ml}$ volume of the mixture was added to a glass beaker containing $300 \mathrm{ml}$ of water under stirring using a magnetic stirrer 
and maintaining the temperature at $37^{\circ} \mathrm{C}$ simultaneously observing emulsion forming tendency. Easy spreading of droplet in water implied good emulsion, whereas oil droplets presence, milky emulsion, or absence of emulsion implied bad emulsion [10].

\section{Development of SNEDDS formulation}

Solubility studies, pseudo-ternary phase diagram, and visual observation formed the basis of SNEDDS formulations of EFV. Peceol as oil phase and Tween 20 and Capmul-MCM as surfactant and co-surfactant, respectively, were used (Table 1). EFV (10 mg) added to accurately weighed amount of oil in screw-capped glass vial was subjected to heating in a water bath at $40^{\circ} \mathrm{C}$ followed by the addition of the surfactant and co-surfactant oily mixture using positive displacement pipette with continuous stirring using a magnetic bar. The storage of formulation was done at room temperature after sonication for $15 \mathrm{~min}$.

\section{Freeze thawing (thermodynamic stability studies)}

Formulations were subjected to thermodynamic stability tests in order for evaluating phase separation and temperature variations effect on SNEDDS formulations. Formulations were subjected to freeze cycle $\left(-20^{\circ} \mathrm{C}\right.$ for 2 days followed by $40^{\circ} \mathrm{C}$ for 2 days). Stable formulations were opted for further studies [11]

\section{Centrifugation}

Phase separation of formulations was observed after centrifuging them for $5 \mathrm{~min}$ at $3000 \mathrm{rpm}$. Formulations stable to phase separation were chosen for further studies [12].

\section{$\%$ Transmittance measurement}

Percentage transmittance of various SNEDDS formulation on reconstitution with distilled water was measured at $248 \mathrm{~nm}$ using UV spectrophotometer against water as a blank [13].

\section{Determination of drug content}

SNEDDS equivalent to $10 \mathrm{mg}$ of EFV was dissolved in $100 \mathrm{ml}$ of Phosphate buffer pH 6.8 after accurate weighing. The drug content was analyzed at $\lambda_{\max } 247 \mathrm{~nm}$ against blank by UV spectrometer after filtration and dilution [14] followed by calculation of actual drug content using the equation specified below:

$\%$ Drug content $=\frac{\text { Actual amount of drug in SNEDDS }}{\text { Theoretical amount of drug in SNEDDS }} \times 100$

\section{In-vitro dissolution studies}

Dissolution studies of SNEDDS of EFV (equivalent to $10 \mathrm{mg}$ of EFV) filled in size " 0 " hard gelatin capsules were performed in US Pharmacopoeia Type II dissolution apparatus with Phosphate buffer pH 6.8, maintaining temperature at $37^{\circ} \mathrm{C}$ and speed at $50 \mathrm{rpm} .5 \mathrm{ml}$ of sample withdrawal was performed at predefined intervals of $2,5,10,15,20,25,30,45$, and $60 \mathrm{~min}$ followed by filtration through $0.45 \mu \mathrm{m}$ pore size membrane filters simultaneously replacing with an equivalent volume of fresh medium buffer at each replacement. The samples were then subjected to spectrophotometric assay at $247 \mathrm{~nm}$.

\section{Characterization of SNEDDS}

Drug-excipient compatibility studies

Compatibility studies between drug and excipients were carried out by Fourier transform infrared (FTIR) spectroscopy method.

\section{FTIR spectroscopy}

The infrared spectra of drug in isotropic mixtures of excipients were obtained by FTIR-8400S Spectrophotometer (Shimadzu, Japan) endowed with attenuated total reflectance accessory, whereas pure drug, i.e., EFV and physical mixtures of the drug with the excipients analysis were done using diffuse reflectance spectroscopy-FTIR with $\mathrm{KBr}$ disc. Residual moisture effect was removed by vacuum drying the samples before obtaining any spectra [15]. Eight scans for each spectrum were obtained at a resolution of $4 \mathrm{~cm}^{-1}$ from a frequency range of 400 to $4000 \mathrm{~cm}^{-1}$.

\section{Determination of droplet size}

Photon correlation spectroscopy was used in the measurement of mean droplet size of EFV SNEDDS formulations by diluting selected formulations with deionized water followed by placement in an electrophoresis cell [16].

\section{Determination of zeta potential}

Zetasizer was used to determine zeta potential of the diluted SNEDDS formulation formed by diluting SNEDDS using distilled water in a ratio of $1: 2500(\mathrm{v} / \mathrm{v})$ with mixing by magnetic stirrer [17]

\section{Scanning electron microscopy (SEM)}

SEM gives a picture of the surface morphology and shape of microspheres. Metal stubs were used for mounting of emulsion that resulted from SNEDDS followed by coating with conductive gold by a sputter coater affixed to the instrument (HITACHI, S-3700N) [18].

\section{Percent entrapment efficiency}

Free drug was separated from emulsion by ultra-filtration at $3500 \mathrm{Da}$ followed by centrifugation at $3000 \mathrm{~g}$ for $5-10 \mathrm{~min}$, then quantifying drug content by high-performance liquid chromatography [19]. The entrapment efficiency was calculated as follows:

$$
\text { Entrapment efficiency }=\frac{\text { Total amount of drug in SNEDDS }}{\text { Total weight of ingredients in emulsion }} \times 100
$$

\section{Stability studies}

Three-month stability tests were conducted at $40^{\circ} \mathrm{C} \pm 2^{\circ} \mathrm{C} / 75 \% \pm 5 \% \mathrm{RH}$ using stability chamber (Thermo Lab, Mumbai) as per the International Council for Harmonization guidelines. At predefined intervals, 0, 30,

Table 1: Formulation trials of liquid self-emulsifying drug delivery systems

\begin{tabular}{|c|c|c|c|c|c|c|}
\hline Smix (Surfactant:Co-surfactant) & Oil:Smix & Formulation code & Drug (efavirenz) (mg) & Oil (Peceol mL) & S-mix (mL) & Water $(\mathrm{mL})$ \\
\hline \multirow[t]{5}{*}{ 1:1 } & 1:9 & F1 & 10 & 0.15 & 1.35 & 0.15 \\
\hline & $2: 8$ & F2 & 10 & 0.3 & 1.2 & 0.3 \\
\hline & $3: 7$ & F3 & 10 & 0.45 & 1.05 & 0.45 \\
\hline & $4: 6$ & $\mathrm{~F} 4$ & 10 & 0.6 & 0.9 & 0.6 \\
\hline & $5: 5$ & F5 & 10 & 0.75 & 0.75 & 0.75 \\
\hline \multirow[t]{5}{*}{$2: 1$} & $4: 6$ & F6 & 10 & 0.6 & 0.9 & 0.6 \\
\hline & $5: 5$ & F7 & 10 & 0.75 & 0.75 & 0.75 \\
\hline & $6: 4$ & F8 & 10 & 0.9 & 0.6 & 1.90 \\
\hline & $7: 3$ & F9 & 10 & 1.05 & 0.45 & 2.00 \\
\hline & $8: 2$ & F10 & 10 & 1.2 & 0.3 & 2.10 \\
\hline \multirow[t]{5}{*}{$3: 1$} & $6: 4$ & F11 & 10 & 0.9 & 0.6 & 3 \\
\hline & $7: 3$ & F12 & 10 & 1.05 & 0.45 & 3.2 \\
\hline & $8: 2$ & F13 & 10 & 1.2 & 0.3 & 4.01 \\
\hline & $9: 1$ & F14 & 10 & 1.35 & 0.15 & 5.2 \\
\hline & $1: 9$ & F15 & 10 & 0.15 & 1.35 & 2.25 \\
\hline
\end{tabular}


60, and 90 days samples were withdrawn. Percent yield, entrapment efficiency, and in-vitro release studies were carried out thereafter [20]

\section{RESULTS AND DISCUSSION}

\section{Solubility studies}

Initially, preliminary solubility studies were conducted for the selection of appropriate excipient from various (Oils - Labrafac PG, Peceol, Acrysol k-150, Capryol PGMC, Oleic acid, and Miglynol 812N; Surfactants - Lauroglycol, Tween 20, Cremophor EL, Kolliphor ELP, and Kolliphor HS 15; and Co-surfactants - PEG 200, Capryol PGE, Brji-35, and Capmul MCM). The solubility of pure drug was $0.009 \mathrm{mg} / \mathrm{mL}$. Peceol, Tween 20, and Capmul MCM were selected as oil, surfactant, and co-surfactant, respectively, on drug solubility basis. The solubility values of drug in these polymers were the highest in comparison to pure drug and other polymers. (Tables 2-4 and Figs. 1-3).

\section{Pseudo-ternary phase diagram}

Peceol, Tween 20, and Capmul MCM were selected as oil, surfactant, and co-surfactant, respectively, by the study of solubility studies. From the ternary phase diagram (Fig. 4), it was observed that self-emulsifying region was enhanced with increasing the concentrations of surfactant and co-surfactant with oil. The efficiency of self-emulsification was good when the surfactant concentration increased.

\section{Table 2: Solubility studies of efavirenz in various oils}

\begin{tabular}{ll}
\hline Oils & Solubility $(\mathbf{m g} / \mathbf{m l})$ \\
\hline Labrafac PG & $198.21 \pm 0.43$ \\
Peceol & $288.12 \pm 0.31$ \\
Acrysol K-150 & $170.51 \pm 0.16$ \\
Capryol PGMC & $93.27 \pm 0.17$ \\
Oleic acid & $42.56 \pm 0.52$ \\
Miglynol 812N & $120.27 \pm 0.48$ \\
\hline
\end{tabular}

Table 3: Solubility studies of efavirenz in various surfactants

\begin{tabular}{ll}
\hline Surfactants & Solubility $(\mathrm{mg} / \mathrm{ml})$ \\
\hline Lauroglycol & $137.2 \pm 0.72$ \\
Cremophor EL & $190.3 \pm 0.43$ \\
Tween 20 & $267.2 \pm 0.62$ \\
Kolliphor ELP & $150.42 \pm 1.11$ \\
Kolliphor HS 15 & $188.22 \pm 2.08$ \\
\hline
\end{tabular}

Table 4: Solubility studies of efavirenz in various co-surfactants

\begin{tabular}{ll}
\hline Co-surfactants & Solubility $(\mathbf{m g} / \mathbf{m l})$ \\
\hline PEG 200 & $235.26 \pm 0.49$ \\
Capryol PGE & $98.37 \pm 0.29$ \\
Brij 35 & $130.59 \pm 0.17$ \\
Capmul MCM & $295.37 \pm 0.17$ \\
Capryol 90 & $220.17 \pm 0.27$ \\
\hline
\end{tabular}

Table 5: Visual observation test for Smix (surfactant: co-surfactant) ratio 1:1

\begin{tabular}{lll}
\hline Oil:Smix & Time of self-emulsification (min) & Grade \\
\hline $1: 9$ & $<1$ & I \\
$2: 8$ & $<1$ & I \\
$3: 7$ & $<1$ & I \\
$4: 6$ & $<1$ & I \\
$5: 5$ & $<1$ & I \\
$6: 4$ & $<1$ & I/II \\
$7: 3$ & $<1$ & I \\
$8: 2$ & $<2$ & III \\
$9: 1$ & $<2$ & III \\
\hline
\end{tabular}

\section{Visual observation}

Emulsion formation tendency was noted by the visual observation method. This test was performed on different formulations prepared by varying ratios of surfactant and co-surfactant ratio (Smix) as 1:1, 2:1, and 3:1. Based on micro-emulsion formation tendency, grades were given to the ratios. Ratios 1:9, 2:8, 3:7, 4:6, and 5:5 of Smix 1:1, 4:6, $5: 5,6: 4,7: 3$, and 8:2 of Smix 2:1, and 6:4, 7:3, 8:2, 9:1, and 1:9 of Smix $3: 1$ showed rapid formation of emulsion within a minute having a clear appearance. Therefore, these ratios were selected for the formulation of SNEDDS. The results are tabulated in Tables 5-7.

\section{Preparation of EFV SNEDDS}

SNEDDS of EFV was formulated using Peceol (Oil), Tween 20 (surfactant), and Capmul MCM (co-surfactant). In the present study, 15 formulations were prepared, and their complete composition is shown in Table 1. All the formulations prepared were found to be clear and transparent. Pictorial representations of formulations F1-F15 are shown in Fig. 5.

\section{Thermodynamic stability studies}

Insignificant phase separation was observed along with negligible varying temperature effects on prepared formulations. Visual

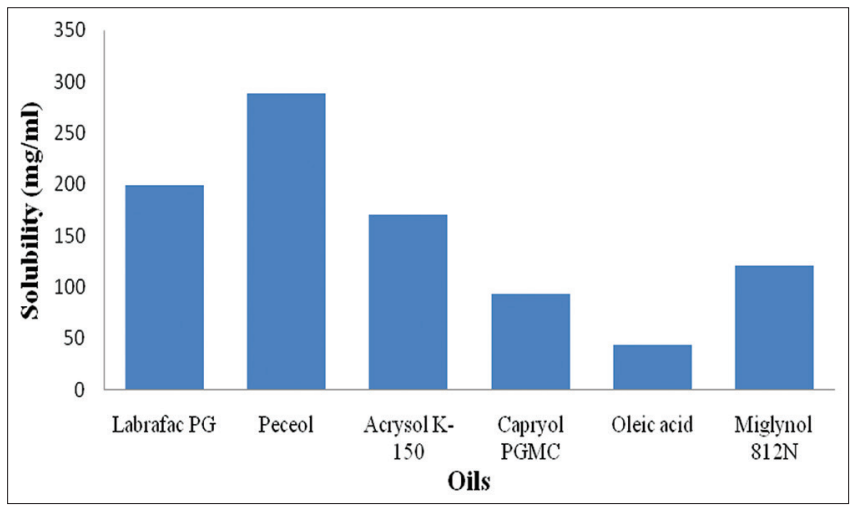

Fig. 1: Solubility studies of efavirenz in oils

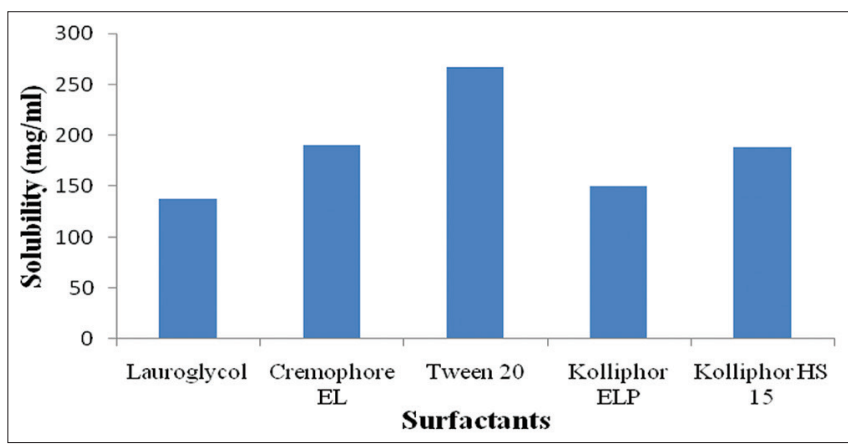

Fig. 2: Solubility studies of efavirenz in surfactant

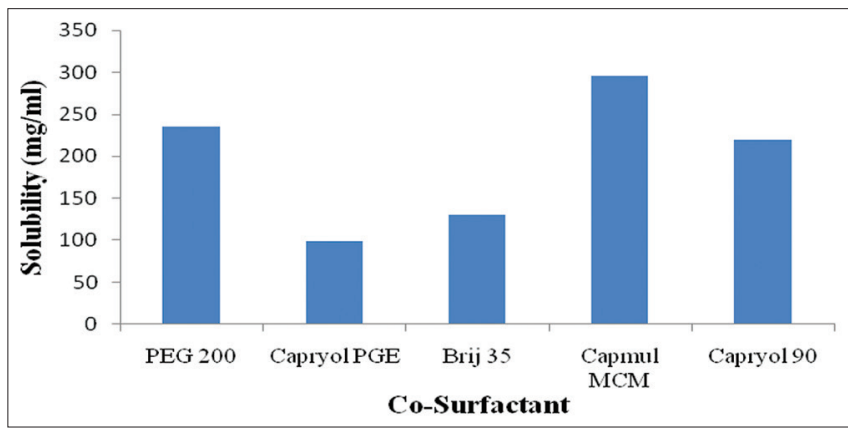

Fig. 3: Solubility studies of efavirenz in co-surfactants 


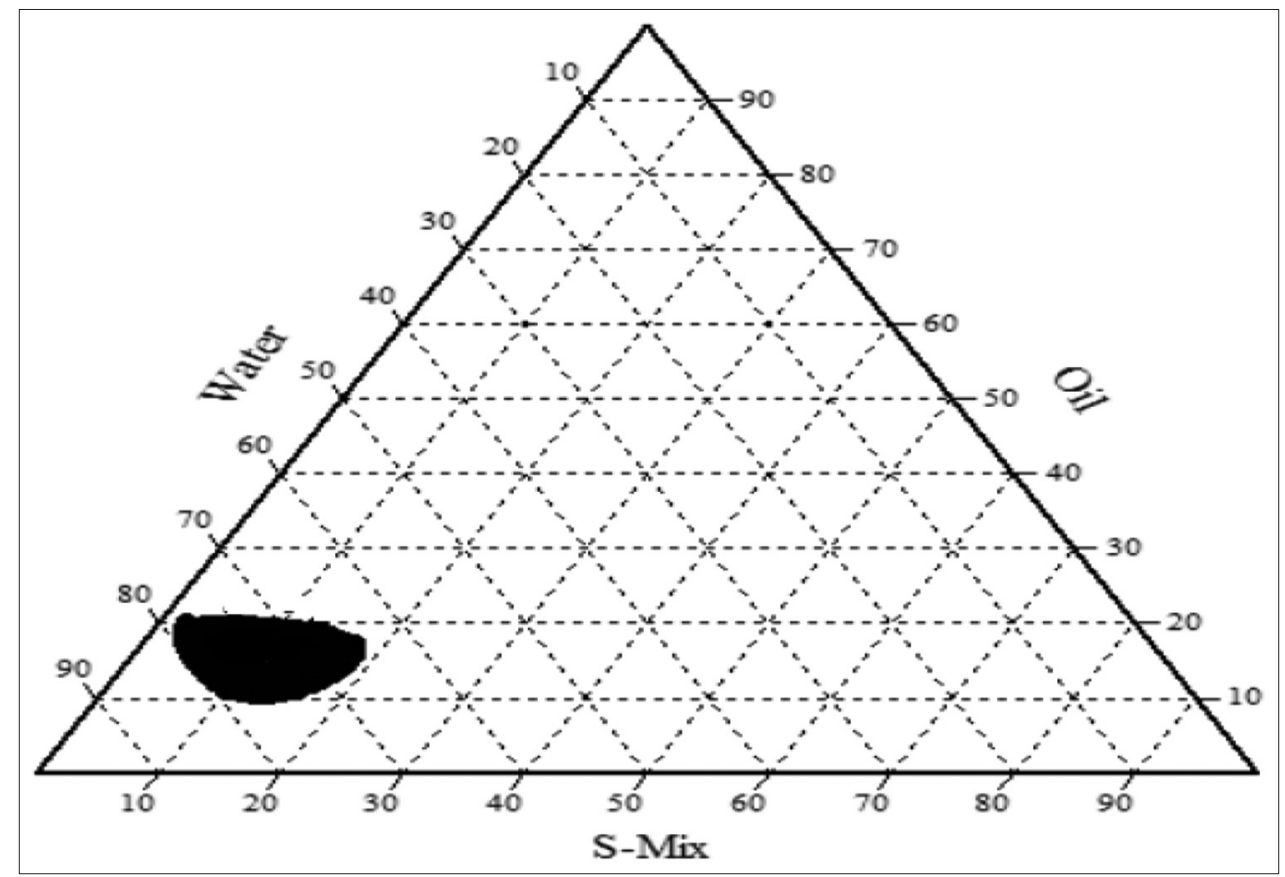

Fig. 4: Ternary phase diagram of peceol, tween 20+Capmul MCM, and water

Table 6: Visual observation test for Smix (surfactant: co-surfactant) ratio $2: 1$

\begin{tabular}{lll}
\hline Oil:Smix & Time of self-emulsification (min) & Grade \\
\hline $1: 9$ & $<1$ & I/II \\
$2: 8$ & $<1$ & I/II \\
$3: 7$ & $<2$ & III \\
$4: 6$ & $<1$ & I \\
$5: 5$ & $<1$ & I \\
$6: 4$ & $<1$ & I \\
$7: 3$ & $<1$ & I \\
$8: 2$ & $<1$ & I \\
$9: 1$ & $<2$ & III \\
\hline
\end{tabular}

Table 7: Visual observation test for Smix (surfactant: co-surfactant) ratio 3:1

\begin{tabular}{lll}
\hline Oil:Smix & Time of self-emulsification (min) & Grade \\
\hline $1: 9$ & $<1$ & I \\
$2: 8$ & $<2$ & III \\
$3: 7$ & $<2$ & III \\
$4: 6$ & $<1$ & I/II \\
$5: 5$ & $<1$ & I/II \\
$6: 4$ & $<1$ & I \\
$7: 3$ & $<1$ & I \\
$8: 2$ & $<1$ & I \\
$9: 1$ & $<1$ & I \\
\hline
\end{tabular}

inspection of samples after centrifugation freeze-thaw cycles indicated no significant changes. Thermodynamically stable formulations were chosen for other characterization (Table 8).

\section{Percent transmittance measurement}

The emulsions were checked for transparency, measured in terms of transmittance (\%T). SNEDDS forms o/w emulsion since water is external phase Formulation F13 has percent transmittance value $>99 \%$ which demonstrates high clarity of emulsion. In general, emulsions exhibiting less clarity have $\mathrm{t}<99 \%$ due to higher globule size that might be the reason for reduction in emulsion transparency and thereby values of $\% \mathrm{~T}$ (Table 9 ).

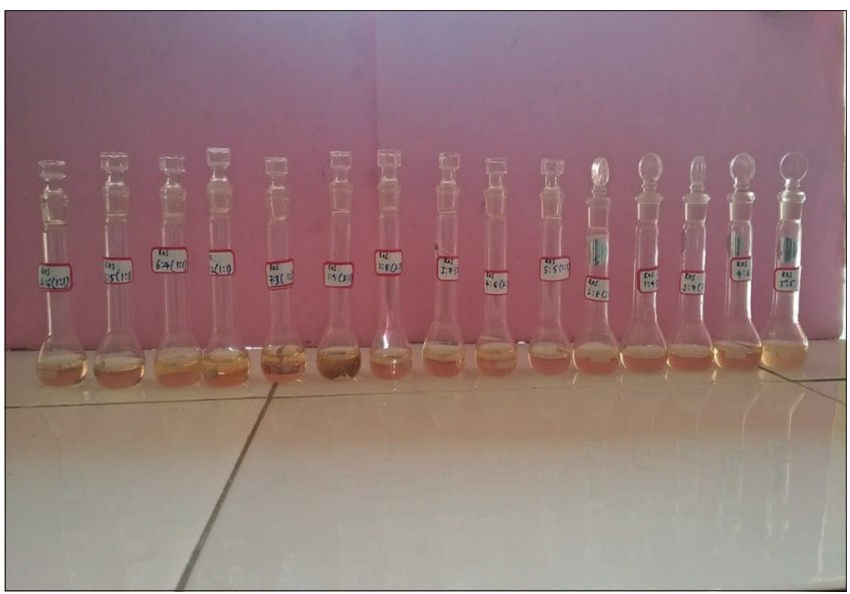

Fig. 5: Formulation No. 1 to No. 15

\section{Drug content of SNEDDS}

Actual drug content of in total 15 formulations is specified in Table 9. The drug content of formulated SNEDDS was in the range of 90.66-98.56\%. A maximum drug release of $98.56 \%$ was noted for formulation F13.

\section{In-vitro dissolution studies of SNEDDS}

Formulation of SNEDDS presents drug in a more solubilize form as droplets that on exposure to the dissolution medium result in rapid drug desolvation. F13 formulation exhibited faster and maximum amount of drug release when compared to other SNEDDS formulations and pure drug clearly illustrating effect of droplet size on drug dissolution rate. (Tables 10-12 and Figs. 6-8)

\section{Interpretation of FTIR data}

The IR spectra of pure drug rosuvastatin showed the presence of principal peaks responsible for different drug excipient interaction is mainly identified by FT-IR spectrums of the both. The wave number $3396.76 \mathrm{~cm}^{-1}$ due to stretching vibration of $\mathrm{O}-\mathrm{H} ; 2856.7 \mathrm{~cm}^{-1}$ due to $\mathrm{C}-\mathrm{H}$ stretching vibrations; $1375.29 \mathrm{~cm}^{-1}$ due to $\mathrm{C}-\mathrm{F}$ stretching vibrations, and $835.21 \mathrm{~cm}^{-1}$ due to $\mathrm{C}=\mathrm{C}$ bending confirm the drug purity. Optimized formulations FTIR spectra had similar fundamental peaks and pattern. 
Table 8: Thermodynamic stability studies of the formulations

\begin{tabular}{lll}
\hline Formulation code & Centrifugation & Freeze-thaw method \\
\cline { 2 - 3 } & & $\mathbf{- 2 0} \mathbf{C}$ for $\mathbf{2}$ days \\
\hline F1 & No phase separation & No change \\
F2 & No phase separation & No change \\
F3 & No phase separation & No change \\
F4 & No phase separation & No change \\
F5 & No phase separation & No change \\
F6 & No phase separation & No change \\
F7 & No phase separation & No change \\
F8 & No phase separation & No change \\
F9 & No phase separation & No change \\
F10 & No phase separation & No change \\
F11 & No phase separation & No change \\
F12 & No phase separation & No change \\
F13 & No phase separation & No change \\
F14 & No phase separation & No change \\
F15 & No phase separation & No change \\
& & No change \\
& & No change \\
No change
\end{tabular}

Table 9: Percentage transmittance of different formulations

\begin{tabular}{lllll}
\hline S. No. & Formulation code & Visual observation & \% Transmittance & \% Drug content \\
\hline 1. & F1 & Transparent & 84.30 & 92.78 \\
2. & F2 & Transparent & 92.14 & 96.44 \\
3. & F3 & Transparent & 90.67 & 95.77 \\
4. & F4 & Slightly clear & 85.37 & 93.12 \\
5. & F5 & Turbid & 65.77 & 97.39 \\
6. & F6 & Transparent & 92.98 & 94.89 \\
7. & F7 & Slightly clear & 75.49 & 94.34 \\
8. & F8 & Slightly clear & 79.67 & 91.27 \\
9. & F9 & Transparent & 94.30 & 90.66 \\
10. & F10 & Slightly clear & 82.77 & 93.48 \\
1. & F11 & Slightly clear & 85.67 & 96.48 \\
12. & F12 & Turbid & 62.79 & 98.56 \\
13. & F13 & Transparent & 98.96 & 93.12 \\
14. & F15 & Slightly clear & 89.63 & \\
15. & & Slightly clear & 90.68 & \\
\hline
\end{tabular}

Table 10: Dissolution profiles of efavirenz self-emulsifying drug delivery systems from F1 to F5

\begin{tabular}{|c|c|c|c|c|c|c|}
\hline \multirow[t]{2}{*}{ Time (min) } & \multicolumn{6}{|c|}{ Dissolution media - phosphate buffer pH 6.8 (\% drug release) formulation code F1-F5 (1:1) } \\
\hline & Pure drug & F1 & F2 & F3 & F4 & F5 \\
\hline 0 & 0 & 0 & 0 & 0 & 0 & 0 \\
\hline 2 & $5.66 \pm 0.07$ & $14.04 \pm 0.90$ & $12.32 \pm 0.85$ & $10.38 \pm 0.84$ & $13.36 \pm 0.86$ & $11.89 \pm 0.84$ \\
\hline 5 & $8.49 \pm 0.59$ & $19.36 \pm 0.99$ & $20.98 \pm 1.10$ & $18.39 \pm 0.98$ & $23.45 \pm 1.20$ & $24.36 \pm 1.21$ \\
\hline 10 & $11.39 \pm 0.85$ & $22.45 \pm 1.15$ & $28.38 \pm 1.45$ & $25.67 \pm 1.19$ & $32.98 \pm 2.37$ & $30.38 \pm 2.35$ \\
\hline 15 & $14.98 \pm 0.90$ & $35.77 \pm 2.36$ & $35.17 \pm 2.36$ & $30.37 \pm 2.32$ & $48.16 \pm 3.32$ & $38.96 \pm 2.36$ \\
\hline 20 & $22.39 \pm 1.15$ & $47.32 \pm 3.32$ & $42.80 \pm 3.34$ & $40.89 \pm 3.32$ & $55.39 \pm 3.99$ & $49.35 \pm 3.33$ \\
\hline 25 & $28.39 \pm 1.45$ & $52.14 \pm 3.98$ & $54.38 \pm 3.99$ & $49.38 \pm 3.34$ & $62.78 \pm 4.08$ & $55.96 \pm 3.99$ \\
\hline 30 & $32.47 \pm 2.25$ & $65.74 \pm 4.02$ & $66.32 \pm 4.08$ & $58.90 \pm 3.99$ & $77.18 \pm 4.22$ & $79.36 \pm 4.22$ \\
\hline 45 & $38.12 \pm 2.98$ & $86.39 \pm 4.38$ & $78.38 \pm 4.23$ & $75.39 \pm 4.20$ & $83.49 \pm 4.92$ & $85.32 \pm 4.36$ \\
\hline 60 & $45.16 \pm 3.15$ & $90.24 \pm 5.01$ & $91.39 \pm 5.01$ & $89.90 \pm 4.99$ & $92.90 \pm 5.02$ & $91.90 \pm 5.01$ \\
\hline
\end{tabular}

Table 11: Dissolution profiles of efavirenz self-emulsifying drug delivery systems from F6 to F10

\begin{tabular}{lllllll}
\hline \multirow{2}{*}{ Time (min) } & \multicolumn{6}{l}{ Dissolution media - Phosphate buffer $\mathbf{p H ~ 6 . 8 ~ ( \% ~ d r u g ~ r e l e a s e ) ~ f o r m u l a t i o n ~ c o d e ~ F 6 - F 1 0 ~ ( 2 : 1 ) ~}$} \\
\cline { 2 - 7 } & Pure drug & F6 & F7 & F8 & F9 & \\
\hline 0 & 0 & 0 & 0 & 0 & 0 & F10 \\
2 & $5.66 \pm 0.07$ & $9.89 \pm 0.80$ & $12.36 \pm 0.86$ & $14.38 \pm 0.94$ & $11.36 \pm 0.85$ & $10.38 \pm 0.81$ \\
5 & $8.49 \pm 0.59$ & $18.36 \pm 0.98$ & $20.98 \pm 1.20$ & $22.36 \pm 1.21$ & $24.38 \pm 1.25$ & $19.45 \pm 0.99$ \\
10 & $11.39 \pm 0.85$ & $24.32 \pm 1.25$ & $28.39 \pm 1.45$ & $31.45 \pm 2.32$ & $35.36 \pm 2.30$ & $26.39 \pm 1.28$ \\
15 & $14.98 \pm 0.90$ & $38.36 \pm 1.89$ & $37.15 \pm 2.98$ & $40.39 \pm 2.98$ & $42.36 \pm 2.98$ & $35.17 \pm 2.30$ \\
20 & $22.39 \pm 1.15$ & $44.12 \pm 2.32$ & $43.18 \pm 3.10$ & $49.47 \pm 3.10$ & $51.32 \pm 3.05$ & $47.38 \pm 3.09$ \\
25 & $28.39 \pm 1.45$ & $56.90 \pm 2.89$ & $58.39 \pm 3.02$ & $52.31 \pm 3.05$ & $60.23 \pm 3.51$ & $54.98 \pm 3.08$ \\
30 & $32.47 \pm 2.25$ & $69.31 \pm 3.58$ & $66.45 \pm 3.58$ & $61.36 \pm 3.50$ & $72.38 \pm 4.08$ & $75.39 \pm 4.09$ \\
45 & $38.12 \pm 2.98$ & $79.36 \pm 4.10$ & $80.39 \pm 4.80$ & $75.56 \pm 4.15$ & $89.47 \pm 4.98$ & $84.38 \pm 4.80$ \\
60 & $45.16 \pm 3.15$ & $89.92 \pm 4.98$ & $91.28 \pm 5.01$ & $90.45 \pm 5.00$ & $92.35 \pm 5.02$ & $93.66 \pm 5.02$ \\
\hline
\end{tabular}


Table 12: Dissolution profiles of efavirenz self-emulsifying drug delivery systems from F11 to F15

\begin{tabular}{|c|c|c|c|c|c|c|}
\hline \multirow[t]{2}{*}{ Time (min) } & \multicolumn{6}{|c|}{ Dissolution media - Phosphate buffer pH 6.8 (\% drug release) formulation code F11-F15 (3:1) } \\
\hline & Pure drug & F11 & F12 & F13 & F14 & F15 \\
\hline 0 & 0 & 0 & 0 & 0 & 0 & 0 \\
\hline 5 & $8.49 \pm 0.59$ & $18.36 \pm 0.98$ & $20.45 \pm 1.20$ & $28.92 \pm 1.45$ & $22.36 \pm 1.21$ & $24.98 \pm 1.25$ \\
\hline 10 & $11.39 \pm 0.85$ & $28.36 \pm 1.45$ & $31.45 \pm 2.32$ & $40.67 \pm 2.98$ & $35.48 \pm 2.32$ & $38.36 \pm 1.89$ \\
\hline 15 & $14.98 \pm 0.90$ & $34.38 \pm 2.32$ & $38.49 \pm 2.98$ & $55.36 \pm 2.89$ & $42.38 \pm 310$ & $45.39 \pm 2.32$ \\
\hline 20 & $22.39 \pm 1.15$ & $42.36 \pm 3.10$ & $49.16 \pm 3.10$ & $67.39 \pm 3.58$ & $54.36 \pm 2.89$ & $58.39 \pm 3.02$ \\
\hline 25 & $28.39 \pm 1.45$ & $53.45 \pm 3.05$ & $55.42 \pm 2.89$ & $72.39 \pm 4.08$ & $62.39 \pm 3.55$ & $65.23 \pm 3.58$ \\
\hline 30 & $32.47 \pm 2.25$ & $64.39 \pm 3.55$ & $68.36 \pm 3.58$ & $80.39 \pm 4.80$ & $70.98 \pm 4.08$ & $72.15 \pm 4.08$ \\
\hline 45 & $38.12 \pm 2.98$ & $79.86 \pm 4.10$ & $81.32 \pm 4.80$ & $89.38 \pm 4.98$ & $85.49 \pm 4.80$ & $88.91 \pm 4.98$ \\
\hline 60 & $45.16 \pm 3.15$ & $89.45 \pm 4.99$ & $91.45 \pm 5.01$ & $98.39 \pm 5.10$ & $94.36 \pm 5.05$ & $93.45 \pm 5.04$ \\
\hline
\end{tabular}

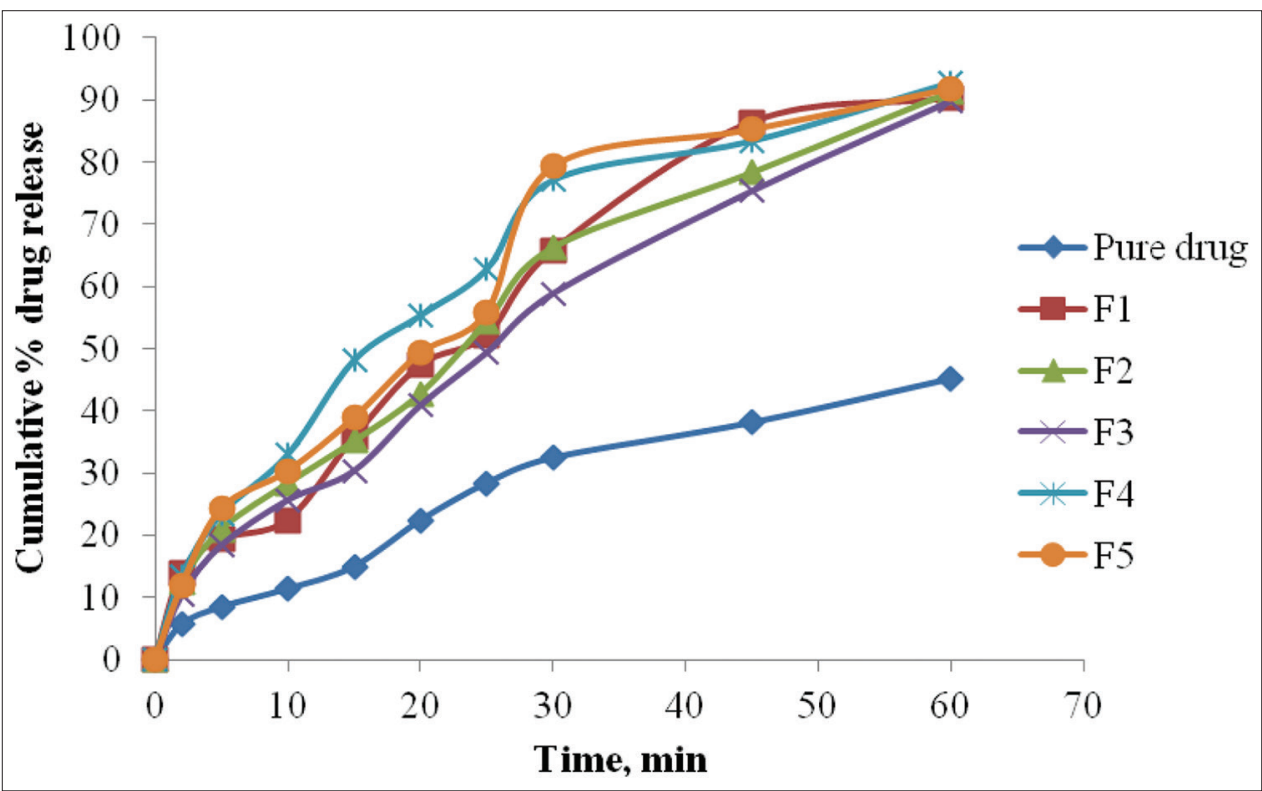

Fig. 6: Dissolution profiles of efavirenz pure drug and formulations (F1 to F5)

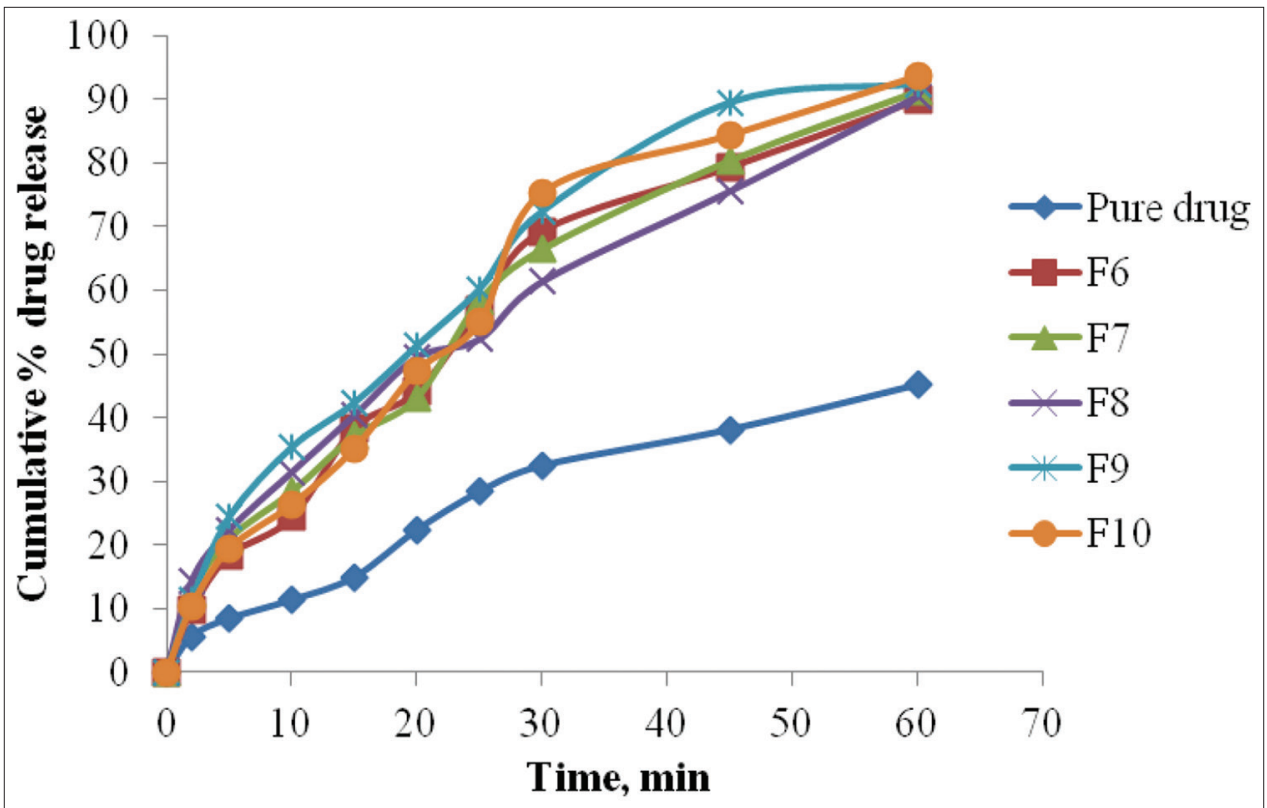

Fig. 7: Dissolution profiles of efavirenz pure drug and formulations (F6 to F10) 


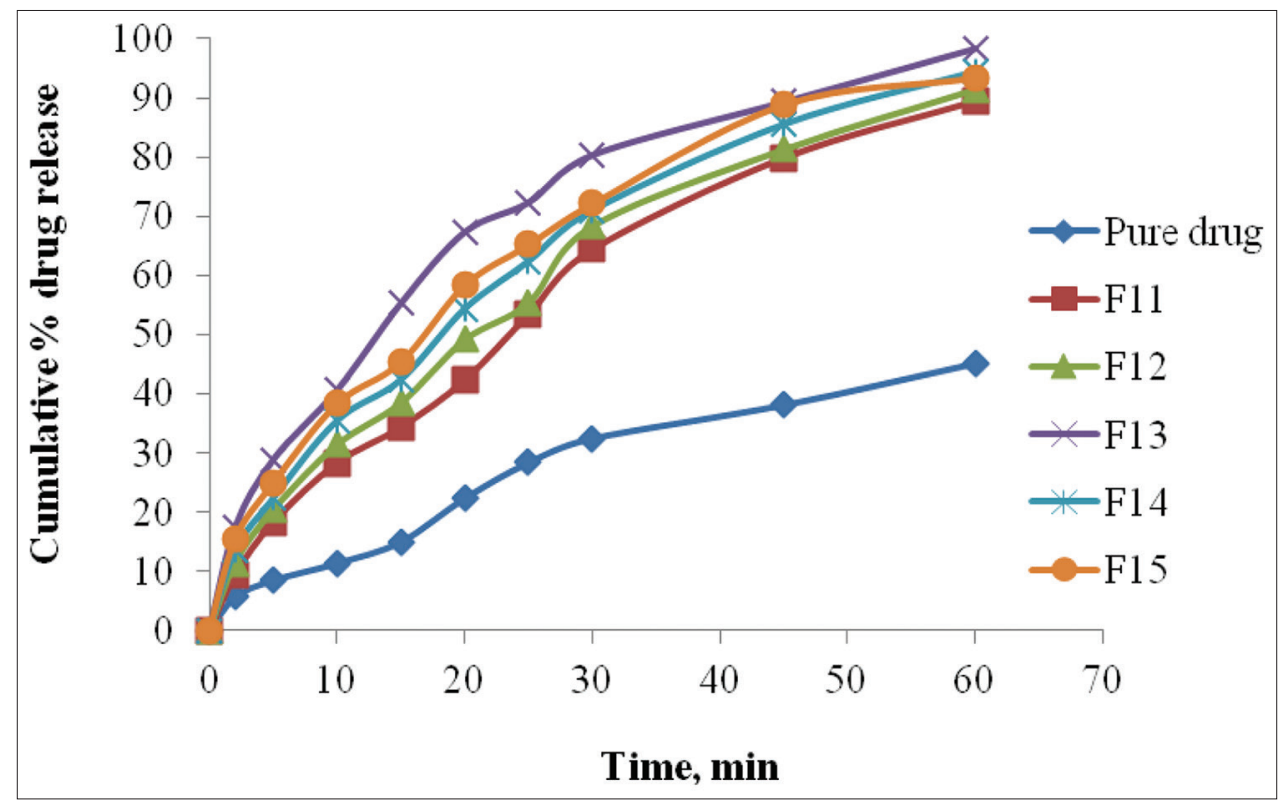

Fig. 8: Dissolution profiles of efavirenz pure drug and formulations (F11 to F15)

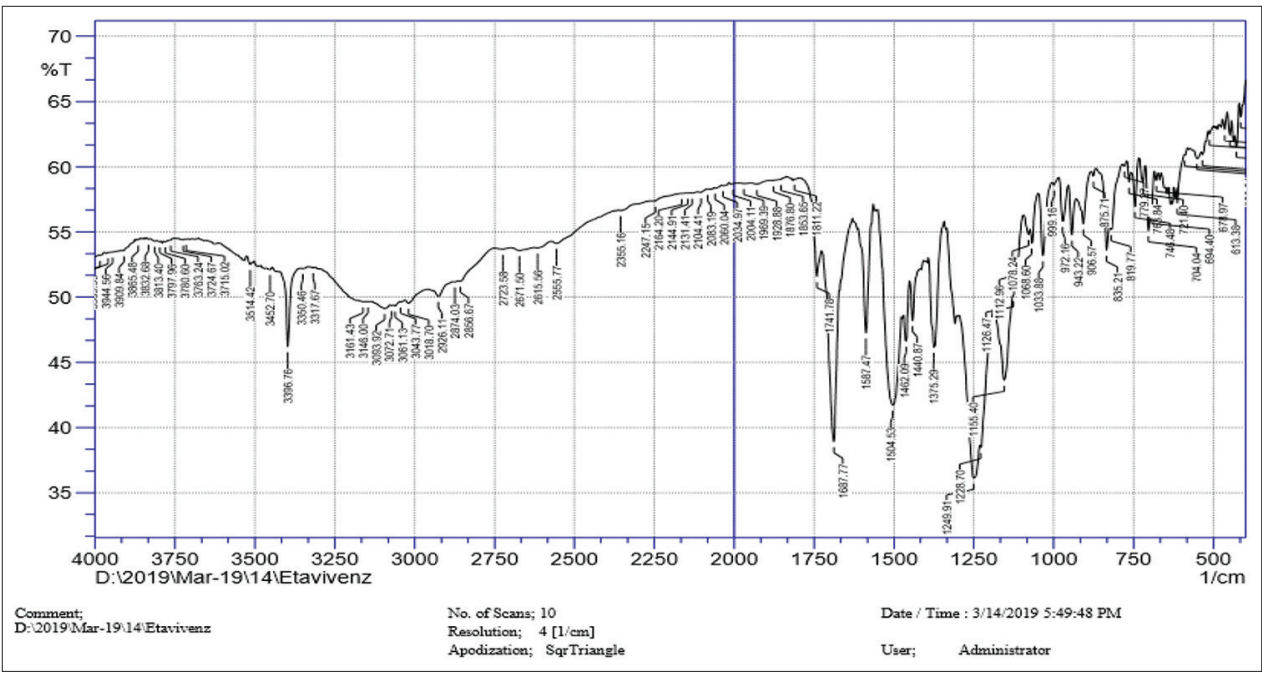

Fig. 9: Fourier transform infrared spectrum of pure drug

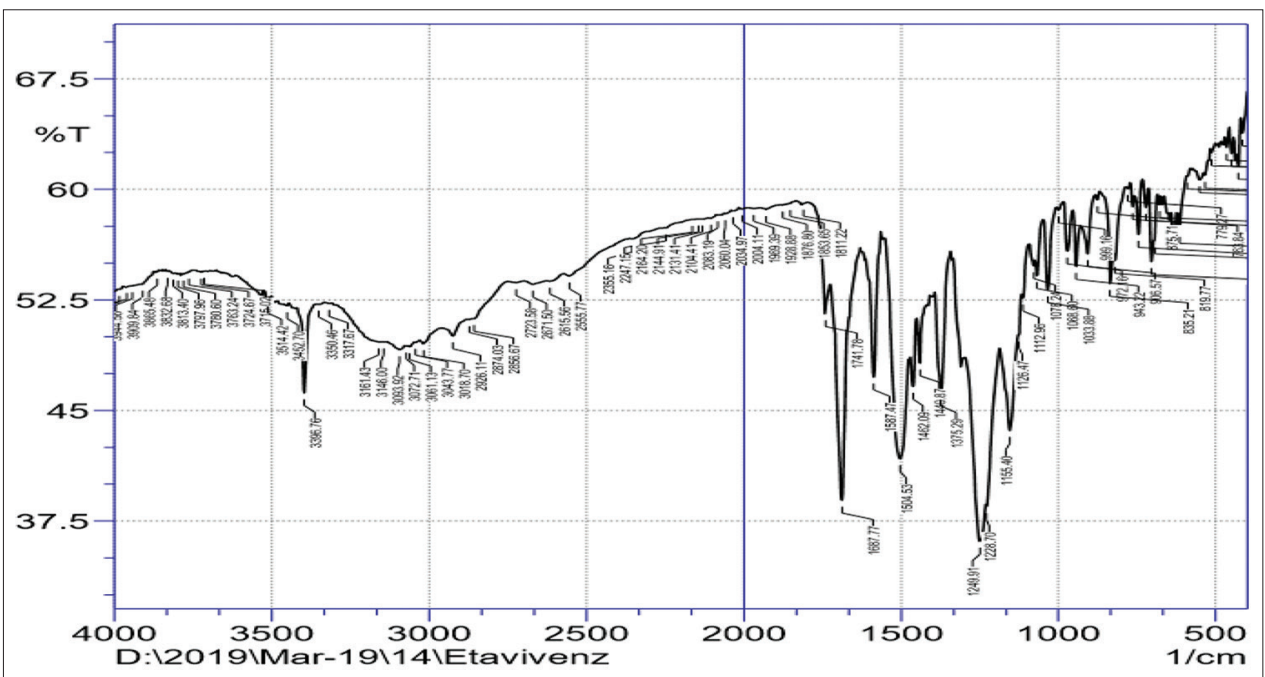

Fig. 10: Fourier transform infrared spectrum of optimized formulation (F13) 


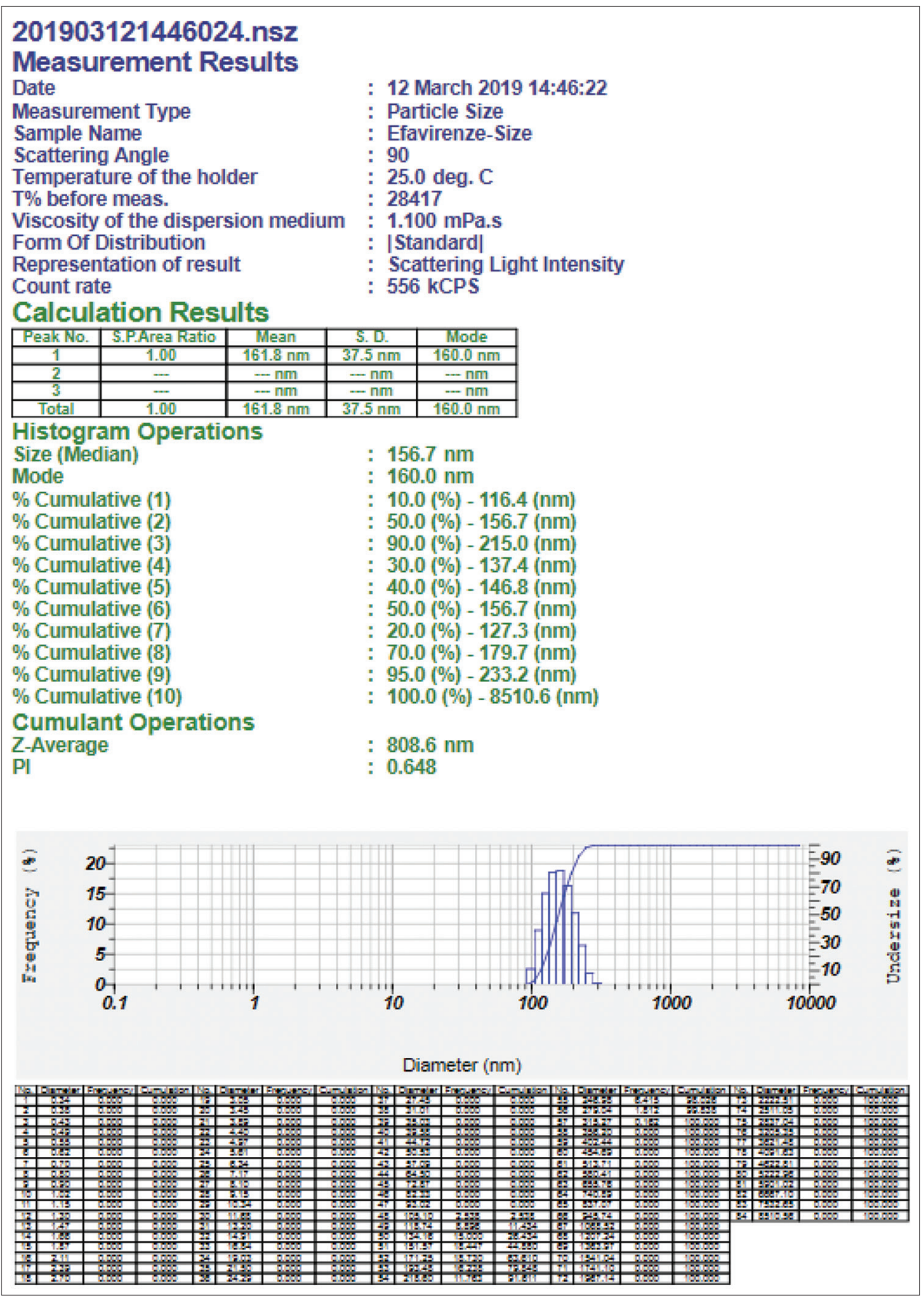

Fig. 11: Particle size analysis of optimized formulation (F13) of efavirenz self-emulsifying drug delivery systems

Thus, there are no significant interactions among the drug and excipients. (Figs. 9 and 10).

\section{Particle size analysis of SNEDDS}

Droplet size is a crucial factor that regulates the drug release rate and extent in addition to absorption profile of the drug. An enhanced bioavailability because of rapid absorption is achieved due to relative increase in interfacial surface area resulting from decreased particle size. An excellent bioavailability has been observed with SNEDDS exhibiting an average droplet size below $200 \mathrm{~nm}$. Optimized SNEDDS formulation had an average droplet was found to be $156.7 \mathrm{~nm}$ and Z-Average of $808.6 \mathrm{~nm}$ with a clear indication of nanometer size ranged droplets (Fig. 11).

\section{Zeta potential of SNEDDS}

Zeta potential is responsible for the extent of repulsion between similar charged, adjacent dispersed droplets. A zeta potential value of $\pm 30 \mathrm{mV}$ is sufficient for the stability of a micro emulsion. Optimized formulation had zeta potential of $-18.3 \mathrm{mV}$ that was in accordance with the zeta potential required for stability (Fig. 12).

\section{SEM for EFV SNEDDS}

SEM studies of optimized formulation (F13) revealed oval-shaped globules. The size is within nanometers. There are clear liquid droplets without any pores (Fig. 13)

\section{Stability studies}

Six months stability study was performed on hard gelatin capsules, filled with EFV SNEDDS F13 formulation. Insignificant change in the release of drug and its contents was observed. There was no significant change in drug content and drug release. The compatibility of formulation with the hard gelatin capsule shells was also noted without any sign of capsule shell deformation. 


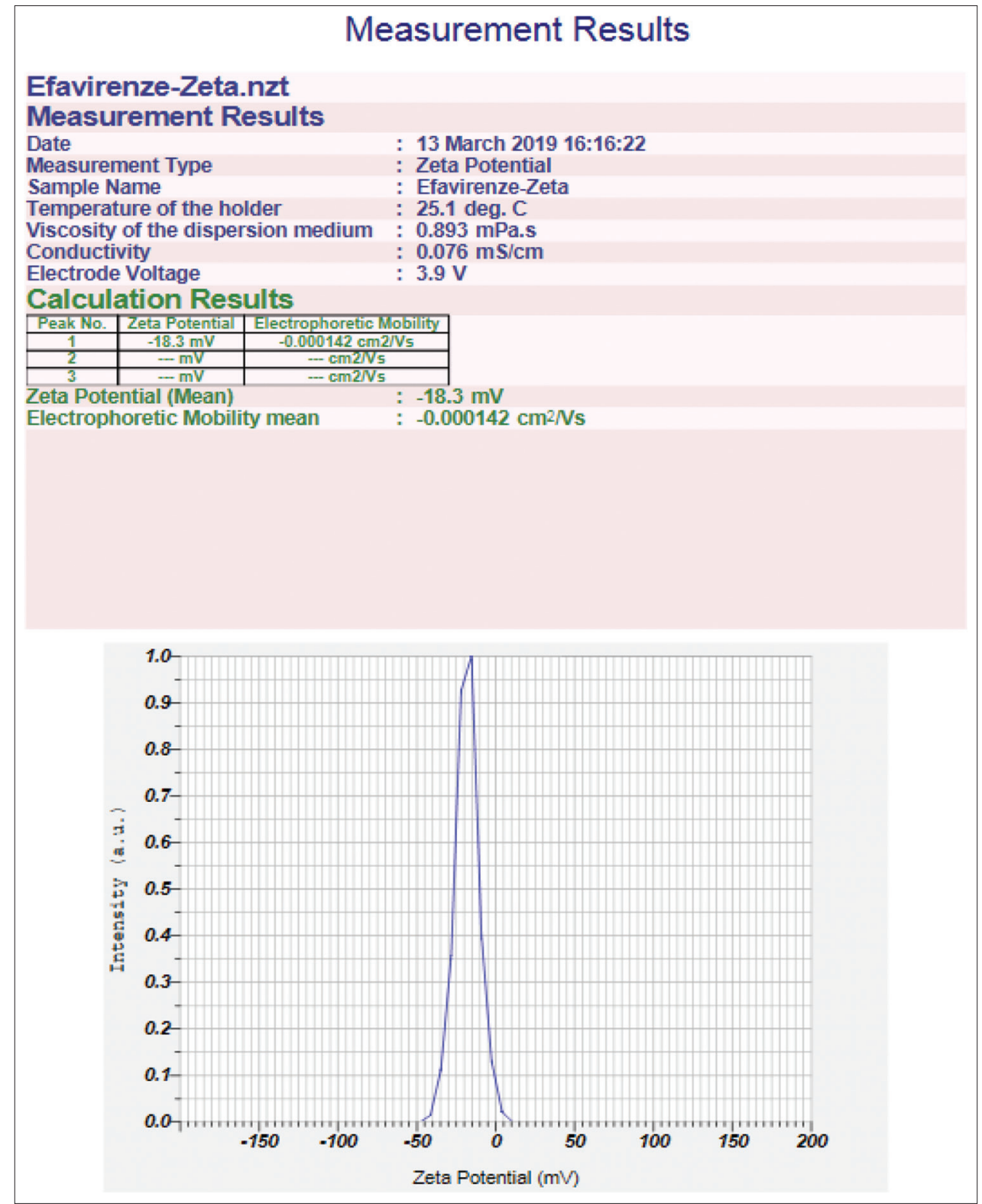

Fig. 12: Zeta potential of the optimized formulation (F13) of efavirenz self-emulsifying drug delivery systems

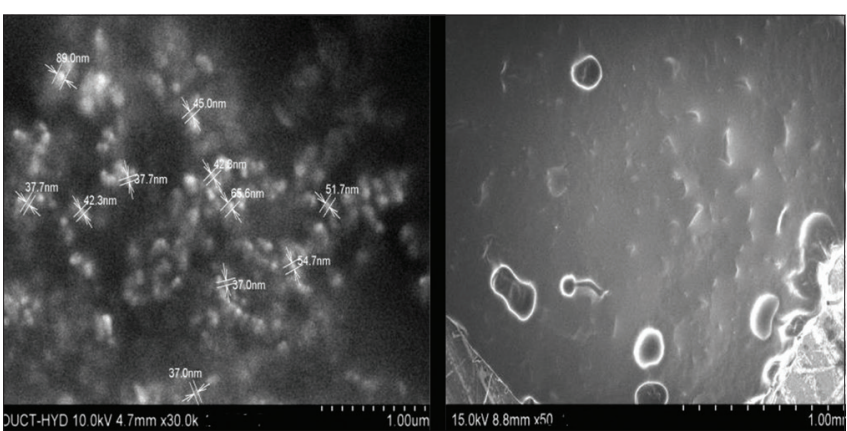

Fig. 13: Scanning electron microscopy of optimized selfemulsifying drug delivery systems formulation

There was negligible change in appearance or microemulsifying property.

\section{SUMMARY AND CONCLUSION}

Different formulations of EFV were formulated using different polymers. From solubility studies, it was observed that EFV showed good solubility in Peceol (Oil), Tween 20 (surfactant), and Capmul MCM which were then chosen as oil, surfactant, and co-surfactant, respectively. Study of pseudo-ternary phase diagram with Peceol, Tween 20, and Capmul MCM as oil, surfactant, and co-surfactant, it was observed that self-emulsifying region was enhanced with increasing concentrations of surfactant and co-surfactant with oil. The drug content of all the formulations was performed with maximum drug content of Formulation F13 that was then selected as optimized one based on other parameters tested. The average droplet size of F13 formulation was $156.7 \mathrm{~nm}$ and Z-Average of $808.6 \mathrm{~nm}$ with clear indication of nanometer ranges particles. The zeta potential of the optimized SNEDDS formulation was found to be $-18.3 \mathrm{mV}$ which was in accordance with the zeta potential required for stability. Thus, this emulsion may serve as a promising alternative approach for the oral delivery of EFV with increased bioavailability.

\section{AUTHOR CONTRIBUTION}

All authors contributed equally.

\section{CONFLICT OF INTEREST}

No conflict of interest by authors. 


\section{REFERENCES}

1. Mistry RB, Sheth NS. A review: Self emulsifying drug delivery system. Int J Pharm Pharma Sci 2011;3:238.

2. Saurabh SS, Issarani R, Nagorib P. Formulation and evaluation of selfemulsifying drug delivery system of etoricoxib. Asian J Pharm Clin Res 2017; 10:367-72.

3. Vijaykumar N, Sandeep K. Development of novel lipid based drug delivery system forraloxifine hydrochloride. Int Res J Pharm 2012;3:2230-8407.

4. PatroSisinthy S, Rao NK, Sarah CL. Design, optimization and In vitro characterization of self nano emulsifying drug delivery system of olmesartan medoxomil. Int J Pharm Sci 2011;3:238

5. Koh PT, Chuah JN, Talekar M, Gorajana A, Garg S. Formulation development and dissolution rate enhancement of efavirenz by solid dispersion systems. Indian J Pharm Sci 2013;75:291-301.

6. Venkateswara Reddy B, Krishna Veni K. Formulation and evaluation of efavirenz microspheres. Der Pharm Lett 2015;7:1-9.

7. Basalious EB, Shawky N, Badr-Eldin SM. SNEDDS containing bioenhancers for improvement of dissolution and oral absorption of lacidipine. I: Development and optimization. Int $\mathrm{J}$ Pharm 2010;391:203-11.

8. Patel J, Kevin G, Patel A, Raval M, Sheth N. Design and development of a self-nanoemulsifying drug delivery system for telmisartan for oral drug delivery. Int J Pharm Investig 2011;1:112-8.

9. Sermkaew N, Ketjinda W, Boonme P, Phadoongsombut N, Wiwattanapatapee R. Liquid and solid self-microemulsifying drug delivery systems for improving the oral bioavailability of andrographolide from a crude extract of Andrographis paniculata. Eur J Pharm Sci 2013;50:459-66.

10. Kaur G, Chandel P, Harikumar SL. Formulation development of self nanoemulsifying drug delivery system (snedds) of celecoxib for improvement of oral bioavailability. Pharmacophore 2013;4:120-33

11. Gupta AK, Mishra DK, Mahajan SC. Preparation and in-vitro evaluation of self-emulsifying drug delivery system of antihypertensive drug Valsartan. Int J Pharm and Life Sci 2011;2:633-9.

12. Bhikshapathi DV, Madhukar P, Dilip KB, Aravind KG. Formulation and characterization of Pioglitazone $\mathrm{HCl}$ self-emulsifying drug delivery system. Sch Res Library 2013;5:292-305.

13. Chirag R. Enhanced oral bioavailability of olmesartan by using novel solid SNEDDS. Int J Adv Pharm 2012;2:82-92.

14. Kapil A, Aggarwal G, Harikumar S. Formulation and evaluation of nano suspension and nano emulsion of rosuvastatin and their comparative study. Asian J Biochem Pharm Res 2015;1:101-16.

15. Nasr V, Gardouh A, Ghorab A. Novel Solid Self-Nano emulsifying drug delivery System (S-SNEDDS) for oral delivery of olmesartan medoxomil: Design formulation, pharmacokinetic and bioavailability evaluation. Pharmaceutics 2016;8:1-29.

16. VanitaSagar S, Subhashini NJ. Self-nano emulsion drug delivery system of fenofibrate with improved bio-availability. Int J Pharm Bio Sci 2013;4:511-21.

17. Date AA, Nagarsenker MS. Design and evaluation of selfnanoemulsifying drug delivery systems (SNEDDS) for cefpodoxime proxetil. Int J Pharm 2007;329:166-72.

18. Ruan G, Feng SS. Preparation and characterization of poly(lactic acid)poly(ethylene glycol)-poly(lactic acid) (PLA-PEG-PLA) microspheres for controlled release of paclitaxel. Biomaterials 2003;24:5037-44.

19. Ke Z, Hou X, Jia XB. Design and optimization of self-nanoemulsifying drug delivery systems for improved bioavailability of cyclovirobuxine D. Drug Des Devel Ther 2016;10:2049-60.

20. Lalit KT, Mohan LK. Stability study and In-vivo evaluation of lornoxicam loaded ethyl cellulose microspheres. Int J Pharm Sci Drug Res 2014;6:26-30. 